\title{
Extraction of Quantitative Information from Non-optimum-focus Aberration- corrected HRTEM Images by Image Processing
}

\author{
C. Wen ${ }^{1,2}$, David J. Smith ${ }^{2}$ \\ 1. School of Science, Southwest University of Science and Technology, Mianyang 621010, China. \\ 2. Department of Physics, Arizona State University, Tempe, Arizona 85287, USA.
}

Aberration-corrected HRTEM images are expected to be taken under the optimum defocus $\left(\Delta f_{\text {opt }}\right)$ condition to correctly reflect the crystal projected structure. However, the corrected state is intrinsically unstable, particularly when acquiring images at a resolution near the microscope information limit [1]. Moreover, the real defocus values are uncertain at the regions of interest away from the specimen edge, where the minimal contrast criterion for Gaussian focus can be applied. These factors can cause the image focus to deviate several nanometers from $\Delta f_{\text {opt }}$. This deviation can have a significant impact on quantitative analysis of HRTEM images, because of image distortions caused by modulation of the contrast transfer function (CTF). For these non-optimum focus images, image processing methods, such as exit-wave reconstruction [2] and image deconvolution [3], can be used to eliminate image distortion and restore the corresponding structure images.

Figure 1 shows the zincblende structure of $3 C$-SiC and its [110] projection. The closely-spaced pairs of atomic columns of C and Si (called "dumbbells") with the separation distance of $1.09 \AA$ are shown in Fig. 1(b). The multislice method was used to calculate series of non-optimum-focus aberration-corrected images for $\mathrm{SiC}$ [110] with varied $\mathrm{Si}$ or $\mathrm{C}$ atomic occupancies and slice thickness of $0.154 \mathrm{~nm}$. The imaging parameters used for calculation were accelerating voltage $U=300 \mathrm{keV}$, instrumental information limit $\delta=0.8 \AA$, specimen thickness $t=1.1 \mathrm{~nm}$ with positive $C_{\mathrm{S}}\left(C_{\mathrm{S}}=10 \mu \mathrm{m}\right.$ and $\left.\Delta f=-8 \mathrm{~nm}\right)$ and negative $C_{\mathrm{S}}\left(C_{\mathrm{S}}=-10 \mu \mathrm{m}\right.$ and $\left.\Delta f=3 \mathrm{~nm}\right)$ conditions, respectively. Figure 2(a) shows the CTF curves for these two conditions comparing with the $\Delta f_{\text {opt }}(\mathrm{mb} \mathrm{nm})$ conditions. Figure 2(b) shows a schematic diagram of ten independent diffraction beams with spatial frequencies below $1.25 \AA^{-1}$. None of the diffracted beams fall at the zero crossovers of the phase CTF curves. After performing deconvolution processing, the structure images restored from the non-optimum-focus images [Figs. 3(a) and (b)] for $\mathrm{SiC}$ [110] with full atomic occupancy are shown in Figs. 3(c) and (d), respectively. All atomic columns appear black and the $\mathrm{SiC}$ dumbbells are clearly visible. The restored structure images for SiC [110] with varied atomic occupancies are shown in Figs. 4(a)-(d). The C or Si occupancy of the center dumbbells (indicated by the hollow arrows) is varied from zero (left image) to full occupancy (right image) in steps of 0.2 , respectively. The image contrast profiles across the center dumbbell positions along the $[00 \overline{1}]$ direction (indicated by the solid arrow) from 0.1 to full $\mathrm{C}$ (or $\mathrm{Si}$ ) occupancy in steps of 0.1 are shown in Figs. 4(e)-(h), respectively. These show that the image contrast of $\mathrm{C}$ (or $\mathrm{Si}$ ) atomic columns increases with the $\mathrm{C}$ (or $\mathrm{Si}$ ) content. Thus, the image processing technique can be regarded as a useful supplementary tool for aberration-corrected microscopes to extract quantitative information on the atomic scale [4].

References:

[1] S.M. Schramm et al, Phys. Rev. Lett. 109 (2012) 163901.

[2] A.I. Kirkland et al, Microsc. Microanal. 10 (2004) 401.

[3] F.H. Li, Phys. Status Solidi A 207 (2010) 2639.

[4] The work was supported by the State Scholarship Fund of China from China Scholarship Council. 


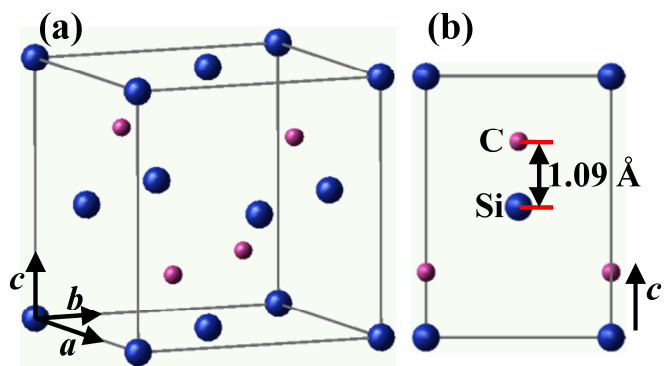

Figure 1. Crystal structure of $3 C$-SiC in perspective view (a), and in projection along the [110] direction (b).
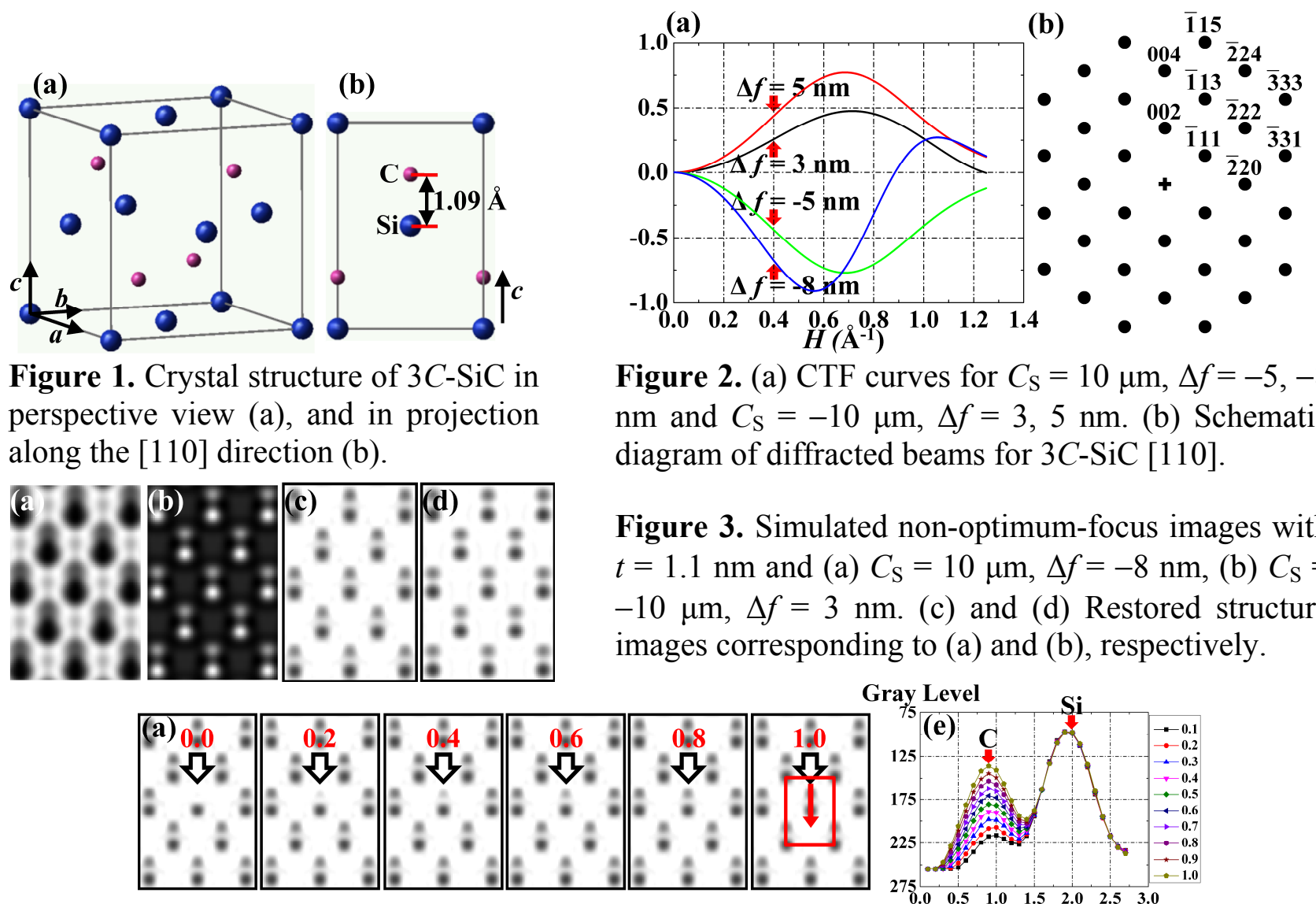

Figure 2. (a) CTF curves for $C_{\mathrm{S}}=10 \mu \mathrm{m}, \Delta f=-5,-8$ $\mathrm{nm}$ and $C_{\mathrm{S}}=-10 \mu \mathrm{m}, \Delta f=3,5 \mathrm{~nm}$. (b) Schematic diagram of diffracted beams for $3 C-\mathrm{SiC}$ [110].

Figure 3. Simulated non-optimum-focus images with $t=1.1 \mathrm{~nm}$ and (a) $C_{\mathrm{S}}=10 \mu \mathrm{m}, \Delta f=-8 \mathrm{~nm}$, (b) $C_{\mathrm{S}}=$ $-10 \mu \mathrm{m}, \Delta f=3 \mathrm{~nm}$. (c) and (d) Restored structure images corresponding to (a) and (b), respectively.
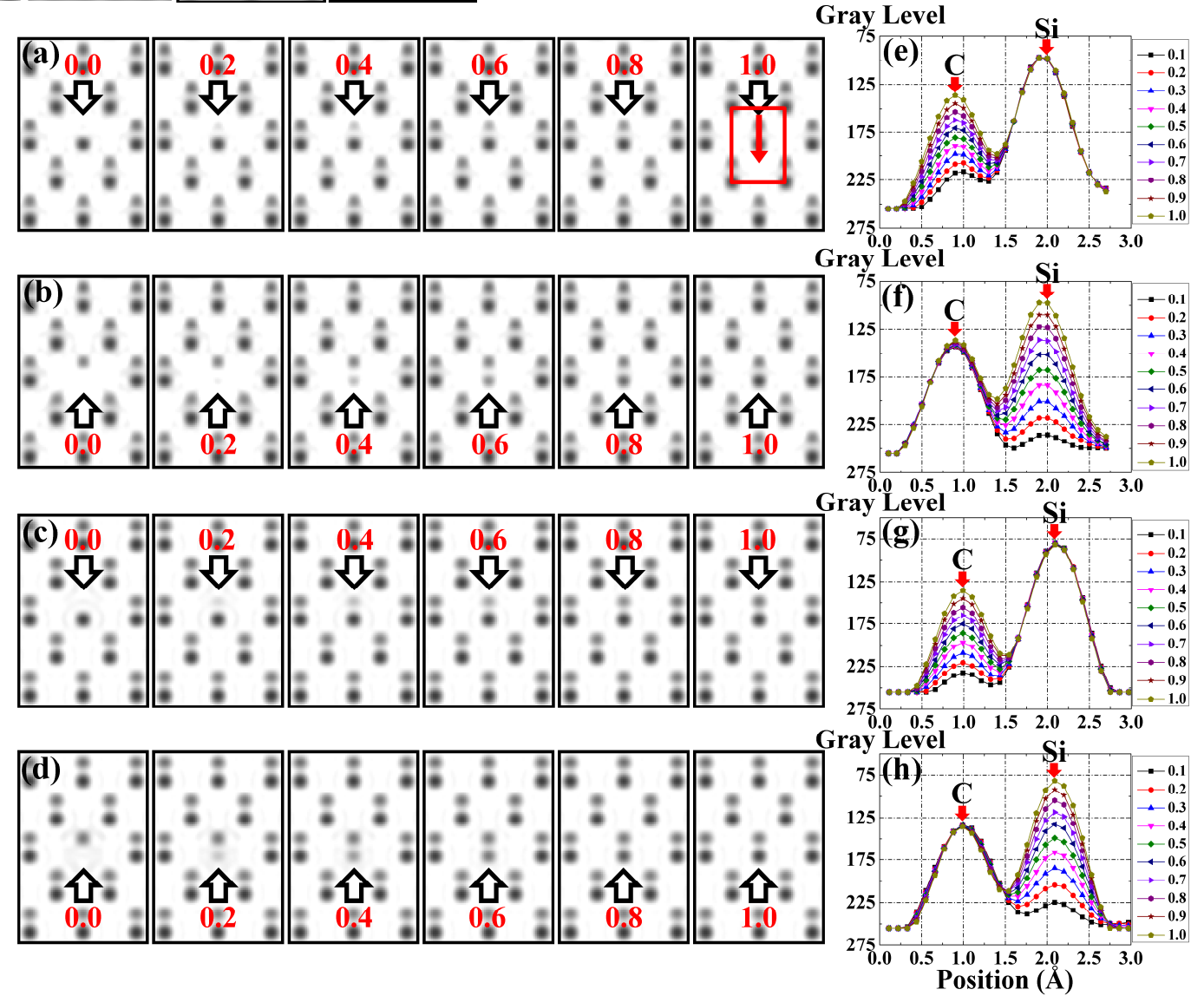

Figure 4. Restored structure images for $\mathrm{SiC}[110]$ with $t=1.1 \mathrm{~nm}, C_{\mathrm{S}}=10 \mu \mathrm{m}, \Delta f=-8 \mathrm{~nm}[(\mathrm{a})$ and (b)] or $C_{\mathrm{S}}=-10 \mu \mathrm{m}, \Delta f=3 \mathrm{~nm}[(\mathrm{c})$ and (d)]. The C [(a) and (c)] or Si [(b) and (d)] occupancy of the center atomic column is varied from zero (left image) to full occupancy (right image) in steps of 0.2. (e)-(h) Image contrast profiles across the atomic columns along [00 $\overline{1}]$ varying from 0.1 to full $\mathrm{C}$ or full Si occupancy in steps of 0.1 , corresponding to (a)-(d), respectively. 